\title{
Análisis de la eficiencia de un amortiguador combinado sintonizado con incertidumbre en los parámetros sometido a una excitación sísmica de alto contenido de frecuencias
}

\author{
Analysis of the efficiency of a combined tuned damper with uncertainty in the parameters \\ subject to a high frequency content seismic excitation \\ Fecha de entrega: 24 de junio 2020 \\ Fecha de aceptación: 28 de octubre 2020
}

\section{Gilda Espinoza, Giordano Sagredo y Álvaro Suazo}

Departamento de Ingeniería Civil y Ambiental, Universidad del Bío-Bío, Avenida Collao 1202, Casilla 5-C, Concepción, Chile, gespinoz@ubiobio.cl,gisagred@alumnos.ubiobio.cl,asuazo@ubiobio.cl

Esta investigación estudia el comportamiento de los parámetros óptimos y de la eficiencia del Amortiguador Combinado Sintonizado (ACS) en el control de una estructura sometida a excitación sísmica, cuando se incorpora incertidumbre en la razón de masa y la razón de longitud. El ACS es un dispositivo compuesto, por un amortiguador de columna de líquido sintonizado (ACLS), y amortiguador de masa sintonizado (AMS). La excitación sísmica es modelada como un proceso estocástico gaussiano de media cero, de tipo estacionario. Se considera una excitación sísmica de alto contenido de frecuencias. Se utiliza el método de linealización estadística equivalente, para linealizar la ecuación no lineal asociada al amortiguador de líquido sintonizado. Se analiza la sensibilidad de los parámetros óptimos, razón de sintonía y coeficiente de pérdida de carga del ACLS, y la razón de sintonía y amortiguamiento del AMS; al incluir una incertidumbre en la razón de masa y en la razón de longitud. Como criterio de optimización se utiliza la reducción del desplazamiento de la estructura principal. Se obtienen la eficiencia del ACS y la raíz media cuadrática RMS (root mean square) del desplazamiento del sistema principal. Los resultados muestran que el parámetro más sensible es el coeficiente de pérdida de carga, ante una incertidumbre en la razón de longitud. Se concluye que la eficiencia del ACS y RMS de desplazamiento de la estructura principal, es insensible a una incertidumbre menor a un 10\%, tanto de la razón de masa y de la razón de longitud.

Palabras clave: ACS, AMS, ACLS, incertidumbre en los parámetros, análisis estocástico
This research studies the behavior of the optimal parameters and the efficiency of the Tuned Combined Damper (TCD) in the control of a structure subjected to seismic excitation, when uncertainty is incorporated in the mass ratio and the length ratio. The TCD is a device made up of a tuned liquid column damper (TLCD), and a tuned mass damper (TMD). Seismic excitation is modeled as a stationary type zeromean Gaussian stochastic process. It is considered a seismic excitation with a high frequency content. The equivalent statistical linearization method is used to linearize the nonlinear equation associated with the tuned fluid damper. The sensitivity of the optimal parameters, the tuning ratio and the head loss coefficient of the TLCD, and the tuning and damping ratio of the AMS are analyzed by including an uncertainty in the mass ratio and the length ratio. Reduction of displacement of the main structure is used as optimization criterion. The TCD efficiency and root mean square RMS of the main system displacement are obtained. The results show that the most sensitive parameter is the head loss coefficient, when faced with an uncertainty in the length ratio. It is concluded that the efficiency of the TCD and RMS of displacement of the main structure is insensitive to an uncertainty of less than 10\%, both of the mass ratio and the length ratio.

Keywords: TCD, TMD, TLCD, parameter uncertainty, stochastic analysis

\section{Introducción y estado del conocimiento}

A través de los años han proliferado las edificaciones en Esto implica que cada vez más, las edificaciones son más altura debido a la escasez de terreno y aumento de población. esbeltas y tienen problemas de grandes desplazamientos. 
Para solucionar esto, han surgido distintos sistemas de reducción de vibraciones.

Entre ellos, están los disipadores de energía y los dispositivos efecto-masa. Entre ellos se encuentran los amortiguadores de masa sintonizados (AMS), los amortiguadores de columna de líquido sintonizado (ACLS) y amortiguadores combinados sintonizado (ACS). Este último consiste en la combinación entre una AMS y un ACLS. El AMS es uno de los dispositivos de control estructural más antiguos, propuesto por primera vez por Frahm (1909). El principio fundamental es que cuando una masa secundaria se une a una masa principal, a través de un resorte, y el sistema principal no tiene amortiguamiento y además la excitación es de tipo armónica, se produce un cero en la función de transferencia y la masa principal no vibra (Den Hartog, 1956). Estudios posteriores analizan el comportamiento de los múltiples AMS (MAMS). Entre estas investigaciones se encuentra la investigación de Singh et al. (2002), quienes proponen como objetivo controlar la reducción de respuestas como corte basal, drift de entre piso, aceleraciones de piso para cuatro AMS.

Posteriormente, Almazán et al. (2012) propusieron el concepto de balance torsional de una estructura asimétrica de un piso con un AMS obteniendo la posición y frecuencia óptimas del AMS que permite reducir la correlación entre el desplazamiento y el giro medido en el centro geométrico de la planta considerando procesos aleatorios. Espinoza et al. (2018a) analizan el comportamiento de un amortiguador de masa sintonizado (AMS) óptimo ubicado en una estructura asimétrica de un piso con tres planos no lineales en dirección de la excitación sísmica. Comparan dos funcionales de optimización, el primero consiste en minimizar el daño en la estructura en todos los planos en forma simultánea. El segundo criterio de optimización consiste en minimizar un funcional de daño, que consiste en un ponderado entre el coeficiente de correlación entre el desplazamiento y giro geométrico de la planta. Luego, Espinoza et al. (2019) investigan la utilización del balance torsional en estructuras asimétricas no lineales usando un enfoque estocástico de la excitación sísmica con el objetivo de evaluar el comportamiento del AMS.

El ACLS consiste en un tubo en forma de U que contiene líquido, generalmente agua. Además, posee un orificio en su parte inferior que provoca una pérdida de carga. Cuando la estructura es sometida a una excitación dinámica, se provoca un movimiento de oscilación del líquido en $\mathrm{su}$ interior, con una frecuencia propia. Este sistema fue propuesto primeramente por Sakai (1989). Después, a partir de una solución cerrada basada en la linealización equivalente y el análisis de vibración aleatoria, Yalla y Kareem (2000) obtienen el coeficiente de pérdida de carga óptimo de un ACLS para un sistema de un grado de libertad no amortiguado sujeto a ruido blanco filtrado.

Di Matteo et al. (2015) proponen una formulación de diseño simplificado para el TLCD bajo carga sísmica estocástica y la validaron experimentalmente mediante una linealización estadística equivalente. Luego, Di Matteo et al. (2017) concluyen que a pesar de que los ACLS producen un rendimiento menor, en comparación con los AMS, poseen facilidad de mantenimiento y son más baratos.

Espinoza et al. (2018b) investigan la eficiencia del ACLS en el control del desplazamiento de la estructura y el daño generado cuando la estructura posee comportamiento no lineal y está sometida a una excitación sísmica estocástica. Wang et al. (2016) proponen un dispositivo que combina un AMS con un ACLS denominado Amortiguador Combinado Sintonizado (ACS). Este dispositivo combina un AMS con un ACLS considerando la eficiencia de un AMS y el fácil manejo y bajo costo del ACLS. Este ACS fue realizado para edificios de gran altura, sometidos a una excitación de viento, que se comportan dentro del rango lineal.

Según Debbarma et al. (2010), la eficiencia de los amortiguadores puede reducirse, si los parámetros no están sintonizados en el modo de vibración para el que está diseñado. Esto implica la presencia inevitable de una incertidumbre en los parámetros del sistema. Cuando se diseña un dispositivo, los parámetros de entrada se consideran exactos. Pero en la realidad, debido a imprecisión en la construcción, en la arquitectura o de algún otro tipo, muchas veces las propiedades cambian.

Por lo tanto, prácticamente no existen investigaciones que incorporen el efecto de la incertidumbre en el análisis de un ACS. Si bien Debbarma et al. (2010) realizan una investigación sobre el comportamiento de los parámetros óptimos de un amortiguador de columna de líquido sintonizado ACLS, cuando incluyen incertidumbre en los parámetros no caracterizan la excitación sísmica, ni analizan la eficiencia. 
Por ello, el objetivo de esta investigación es analizar el comportamiento de los parámetros de diseño de un CTD, y la eficiencia en el control de una estructura sometida a una excitación sísmica de alto contenido de frecuencias cuando existe incertidumbre en los parámetros del sistema. El caso de una excitación sísmica de bajo contenido de frecuencias con incertidumbre es abordado por Espinoza et al. (2020).

\section{Modelo estructural}

La Figura 1 muestra el modelo de estudio (M1), que consiste en una estructura lineal, a la que se le ha añadido un CTD (combined tuned damper), que consiste en un AMS y un ACLS. La estructura principal consiste en una masa $M_{s}$, que posee una rigidez $k_{s}$, y un coeficiente de amortiguamiento $C_{s}$, la que está sometida a una excitación basal sísmica $\ddot{u}_{g}$. Adherida a la estructura principal, se encuentra un AMS que consiste en una masa $M_{T}$, unida a la estructura principal, a través de un resorte de rigidez $k_{T} \mathrm{y}$ un amortiguador viscoso con una constante de amortiguación $C_{T}$. En el ACLS, $B_{h}$ y $h$ son las dimensiones horizontal y vertical del sistema ACLS, respectivamente.

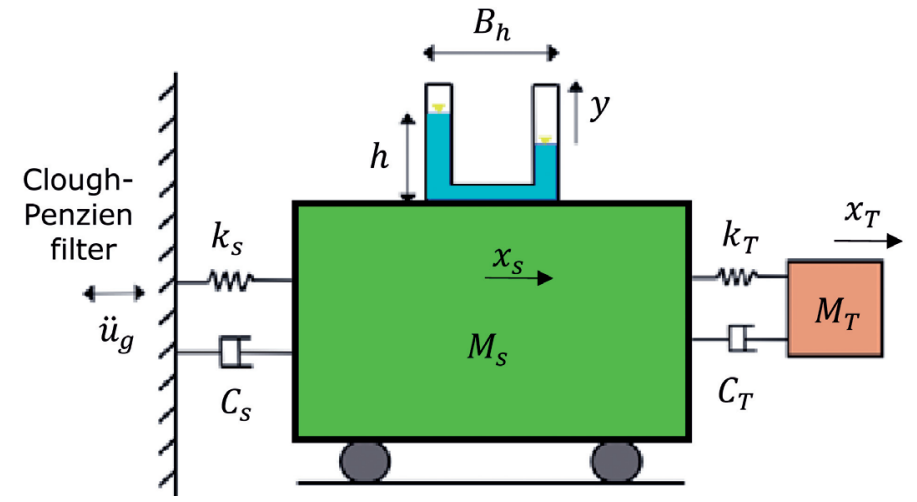

Figura 1: Estructura principal con CTD. Modelo1 (M1)

M1 está sometido a una aceleración sísmica estocástica en la base de la estructura principal, resultante del filtrado de una señal de ruido blanco en la roca.

\section{Formulación dinámica de M1}

El vector de grados de libertad (gdl) de M1, se muestra en (1):

$$
\mathbf{X}=\left\{\begin{array}{lll}
x_{S}(t) & x_{T}(t) & y(t)
\end{array}\right\}^{T}
$$

donde $x_{s}$ representa el desplazamiento de la estructura principal en dirección $x, x_{T}$ representa el desplazamiento del AMS en dirección $x$, e $y$ indica el desplazamiento de la superficie del líquido dentro del ACLS en sentido vertical. El equilibrio dinámico del ACLS viene dado por (Sakai, 1989):

$$
\begin{aligned}
& \left.\rho A L_{e} \ddot{y}+2 \rho A \xi_{l} \mid \dot{y}\right\rfloor \dot{y}+2 \rho g A y=-\rho A B_{h}\left(\ddot{x}_{s}+\ddot{u}_{g}\right) \\
& L_{e}=B_{h}+2 h
\end{aligned}
$$

donde $\rho$ es la densidad del líquido, $A$ es la sección transversal, $L_{e}=B_{h}+2 h$ es la longitud total del ACLS, $\xi_{l}$ es el coeficiente de pérdida de carga del ACLS, $g$ es la aceleración de gravedad, $\ddot{x}_{s}$ y $\ddot{u}_{g}$ son la aceleración de la estructura principal y de la base. La expresión (2) se puede linealizar estadísticamente a través de la inclusión de un amortiguamiento lineal equivalente, donde $\sigma_{\dot{y}}$ es la desviación estándar de la velocidad del líquido.

$$
C_{P}=\frac{\xi_{l} \sigma_{\dot{y}}}{\sqrt{2 \pi}}
$$

Debido a que $\mathrm{M} 1$ posee $3 \mathrm{gdl}$, las ecuaciones de equilibrio dinámico necesarias para modelar dinámicamente son tres:

$$
\begin{aligned}
& \left(M_{S}+M_{T}+M_{L}\right)\left(\ddot{x}_{s}+\ddot{u}_{g}\right)+M_{T} \ddot{x}_{T}+M_{H L} \ddot{y}+ \\
& C_{S} \dot{x}_{s}+k_{s} x_{s}=0 \\
& M_{T}\left(\ddot{x}_{T}+\ddot{x}_{s}+\ddot{u}_{g}\right)+C_{T} \dot{x}_{T}+k_{T} x_{T}=0 \\
& M_{L} \ddot{y}+M_{H L}\left(\ddot{x}_{s}+\ddot{u}_{g}\right)+C_{L} \dot{y}+k_{L} y=0
\end{aligned}
$$

donde, $M_{L}=\rho A L_{e}$ es la masa del líquido, $M_{H L}=\rho A B_{h}$ es la masa horizontal del líquido, $C_{L}=2 \rho A C_{p}$ es el coeficiente de amortiguamiento asociado al líquido y $k_{L}=2 \rho g A$ es la rigidez asociada al líquido. Como se observa, (4), (5) y (6) son ecuaciones diferenciales de segundo orden acopladas, donde (4) y (5) son ecuaciones lineales y (6) es una ecuación linealizada originalmente no lineal.

\section{Formulación en espacio de estado}

Las ecuaciones (4), (5) y (6) se normalizan respecto a la masa de la estructura principal $M_{s}$. Considerando las relaciones (7a) y (7b) para la estructura principal:

$\frac{C_{s}}{M_{s}}=2 \xi_{s} \omega_{s} \quad ; \quad \frac{k_{s}}{M_{s}}=\omega_{s}^{2}$

Para el AMS, se considera la razón de masa del AMS y la masa de la estructura principal $\left(\mu_{T}\right)$ con $(7 a)$ y $(7 b)$, para obtener: 


$$
\begin{aligned}
& \frac{M_{T}}{M_{S}}=\mu_{T} \quad ; \quad \frac{C_{T}}{M_{S}}=\frac{2 \xi_{T} \omega_{T} M_{T}}{M_{S}}=2 \xi_{T} \omega_{T} \mu_{T} \quad ; \\
& \frac{k_{T}}{M_{S}}=\frac{\omega_{T}^{2} M_{T}}{M_{S}}=\omega_{T}^{2} \mu_{T}
\end{aligned}
$$

En el caso del ACLS, se define $\mu_{L}$ como la razón de masa entre el amortiguador del líquido y la estructura principal y $p$ como la razón de longitud:

$$
\mu_{L}=\frac{M_{L}}{M_{S}} \quad ; \quad p=\frac{B_{h}}{L_{e}}
$$

Luego se estipulan las siguientes igualdades:

$$
\begin{aligned}
& \frac{M_{L H}}{M_{S}}=\mu_{L} p \quad ; \quad \frac{C_{L}}{M_{S}}=\frac{2 C_{P} \mu_{L}}{L_{e}} \quad ; \\
& \frac{k_{L}}{M_{S}}=\frac{2 g \mu_{L}}{L_{e}} ; L_{e}=\frac{2 g}{\omega_{l}^{2}}
\end{aligned}
$$

Formulando las ecuaciones (4), (5) y (6) normalizadas y en forma matricial queda:

$$
\begin{aligned}
& \mathbf{M} \ddot{\mathbf{X}}+\mathbf{C} \dot{\mathbf{X}}+\mathbf{K X}=\mathbf{B}_{\mathbf{u}} \ddot{u}_{g} \\
& \mathbf{M}=\left[\begin{array}{ccc}
1+\mu_{T}+\mu_{L} & \mu_{T} & \mu_{L} p \\
\mu_{T} & \mu_{T} & 0 \\
\mu_{L} p & 0 & \mu_{L}
\end{array}\right] \mathbf{C}=\left[\begin{array}{ccc}
2 \xi_{S} \omega_{S} & 0 & 0 \\
0 & 2 \xi_{T} \omega_{T} \mu_{T} & 0 \\
0 & 0 & 2 \frac{C_{P} \mu_{L}}{L_{e}}
\end{array}\right] \\
& \mathbf{K}=\left[\begin{array}{ccc}
\omega_{S}^{2} & 0 & 0 \\
0 & \mu_{T} \omega_{T}^{2} & 0 \\
0 & 0 & \frac{2 g \mu_{L}}{L_{e}}
\end{array}\right] \quad \mathbf{B}_{\mathbf{u}}=\left[\begin{array}{c}
-\left(1+\mu_{T}+\mu_{L}\right) \\
-\mu_{T} \\
-\mu_{L} p
\end{array}\right]
\end{aligned}
$$

Lo que en espacio de estado se expresa como:

$$
\begin{aligned}
& \dot{\mathbf{X}}=\mathbf{A} \mathbf{X}+\mathbf{B} \ddot{u}_{g} \\
& \mathbf{A}=\left[\begin{array}{cc}
\mathbf{0}_{3 \times 3} & \mathbf{I}_{3 \times 3} \\
-\mathbf{M}^{-1} \mathbf{K} & -\mathbf{M}^{-1} \mathbf{C}
\end{array}\right] \\
& \mathbf{B}=\left\{\begin{array}{c}
\mathbf{0} \\
-\mathbf{M}^{-1} \mathbf{1}_{3 \times 3}
\end{array}\right\}
\end{aligned}
$$

\section{Filtro de Clough-Penzien}

El movimiento del suelo se genera desde una señal de ruido blanco en la roca, filtrada por el estrato de suelo por un doble filtro de Clough-Penzien (Clough y Penzien, 1975), llegando como una aceleración a nivel de base de la estructura. En este estudio el input considerado es una excitación estocástica estacionaria de ancho de banda amplia, en inglés Broad Band Process (BBP), es decir, de alto contenido de frecuencias. Cuando se realiza la transformada de Fourier a una señal, en este caso una excitación sísmica, en la gráfica se puede observar la potencia asociada a cada frecuencia que contiene la señal. Las frecuencias que tienen mayor potencia son las más importantes en la representación de la señal. Si la primera frecuencia de alta importancia y la última de alta importancia están muy separadas, se llaman de ancho de banda amplio, es decir, se necesita un gran contenido de frecuencias para caracterizar la excitación sísmica. El filtro se utilizó para definir la Densidad de Potencia Espectral (Power Spectral Density, PSD). Para ello se ajustó el filtro de Clough-Penzien a la PSD obtenida de un sismo artificial compatible con la norma chilena NCh2745 (2013), para zona sísmica 2 y suelo tipo B, a partir de una aproximación por mínimos cuadrados. Esto determina un input de BBP, por la naturaleza de alto contenido de frecuencias de los sismos chilenos. Entonces la función PSD es $S_{g}(\omega)$ del movimiento del suelo:

$$
\begin{aligned}
& S_{g}(\omega)=S_{o} \frac{\omega_{g}+4 \xi_{g}^{2} \omega_{g}^{2} \omega^{2}}{\left(\omega_{g}^{2}-\omega^{2}\right)^{2}+4 \xi_{g}^{2} \omega_{g}^{2} \omega^{2}} . \\
& \frac{\omega^{4}}{\left(\omega_{f}^{2}-\omega^{2}\right)^{2}+4 \xi_{f}^{2} \omega_{f}^{2} \omega^{2}}
\end{aligned}
$$

donde $S_{o}, \omega_{g}, \xi_{g}, \omega_{f}$ y $\xi_{f}$ representan la intensidad de ruido blanco, frecuencia del estrato de suelo, razón de amortiguamiento del estrato de suelo, frecuencia del filtro y razón de amortiguamiento del filtro, respectivamente. Los valores utilizados se muestran en la Tabla 1.

Tabla 1: Parámetros del filtro de Clough - Penzien

\begin{tabular}{|c|c|c|c|c|}
\hline$S_{o}$ & $\omega_{g}, \mathrm{rad} / \mathrm{s}$ & $\xi_{g}$ & $\omega_{f}, \mathrm{rad} / \mathrm{s}$ & $\xi_{f}$ \\
\hline 1335.6 & 16.57 & 0.491 & 3.02 & 0.48 \\
\hline
\end{tabular}

\section{Extensión del estado para inclusión del filtro}

Para incluir el filtro, se plantea el siguiente vector de estado del filtro (Saitua et al., 2018):

$$
\mathbf{X}_{\mathbf{f}}=\left\{\begin{array}{llll}
X_{g} & \dot{X}_{g} & X_{f} & \dot{X}_{f}
\end{array}\right\}^{T}
$$

donde $X_{g}, \dot{X}_{g}$ representan el desplazamiento y velocidad del suelo, mientras que $X_{f}, \dot{X}_{f}$, el desplazamiento y velocidad del filtro. La ecuación de estado del filtro se expresa como: 


$$
\dot{\mathbf{X}}_{\mathbf{f}}=\mathbf{A}_{\mathbf{f}} \mathbf{X}_{\mathbf{f}}+\mathbf{B}_{\mathbf{f}} W(t) \quad \ddot{u}_{g}=\mathbf{C}_{\mathbf{f}} \mathbf{X}_{\mathbf{f}}
$$

donde $A_{f}$ es la matriz de estado del filtro, $B_{f}$ es la matriz de colocación del filtro y $C_{f}$ es la matriz del output del filtro, las cuales vienen dadas por:

$$
\begin{aligned}
\mathbf{A}_{\mathbf{f}} & =\left[\begin{array}{cccc}
0 & 1 & 0 & 0 \\
-\omega_{g}^{2} & -2 \xi_{g} \omega_{g} & 0 & 0 \\
0 & 0 & 0 & 1 \\
\omega_{g}^{2} & 2 \xi_{g} \omega_{g} & -\omega_{f}^{2} & -2 \xi_{f} \omega_{f}
\end{array}\right] \quad \mathbf{B}_{\mathbf{f}}=\left[\begin{array}{c}
0 \\
-1 \\
0 \\
0
\end{array}\right] \\
\mathbf{C}_{\mathbf{f}} & =\left\{\begin{array}{llll}
\omega_{g}^{2} & 2 \xi_{g} \omega_{g} & -\omega_{f}^{2} & -\xi_{f} \omega_{f}
\end{array}\right\}
\end{aligned}
$$

Para incluir el filtro en la formulación dinámica de M1, se debe aumentar el estado, para lo cual se definió el vector de estado aumentado. Considerando el vector de excitación:

$$
\begin{aligned}
& \mathbf{X}_{2}=\left\{\begin{array}{lllllll}
x_{s}(t) & x_{T}(t) & y(t) & \dot{x}_{S}(t) & \dot{x}_{T}(t) & \dot{y}(t) & x_{g}(t) \\
\dot{X}_{g}(t) & X_{f}(t) & \dot{X}_{f}(t)
\end{array}\right\}^{T}
\end{aligned}
$$

Extendiendo la ecuación de estado Estructura-AMS-ACLS a Estructura-AMS-ACLS-FILTRO queda:

$$
\dot{\mathbf{X}}_{2}=\mathbf{A}_{2} \mathbf{X}_{2}+\mathbf{B}_{2} W(t)
$$

De lo que se obtiene finalmente:

$$
\mathbf{A}_{2}=\left[\begin{array}{cc}
\mathbf{A} & \mathbf{B C}_{\mathbf{f}} \\
0 & \mathbf{A}_{\mathbf{f}}
\end{array}\right] \quad \mathbf{B}_{2}=\left[\begin{array}{c}
0 \\
\mathbf{B}_{\mathrm{f}}
\end{array}\right]
$$

donde $\mathbf{A}_{2}$ corresponde a la matriz y $\mathbf{B}_{2}$ al vector de excitación del sistema completo.

\section{Cálculo de matriz de covarianza}

Para el cálculo de la matriz de covarianza se resolverá la ecuación de Lyapunov (24b). Para calibrar la intensidad del ruido blanco, se consideró una varianza de aceleración del suelo en la entrada de la estructura como:

$$
\sigma_{\ddot{u}_{g}}^{2}=\frac{P G A}{3} \quad ; \quad \mathbf{A}_{\mathbf{2}} \mathbf{R}+\mathbf{R} \mathbf{A}_{2}^{\mathrm{T}}+\mathbf{B}_{2} S_{o} \mathbf{B}_{2}^{\mathrm{T}}=0
$$

donde $P G A$ es el peak ground acceleration considerado, correspondiente a $0.3 \mathrm{~g}$ para este estudio. $\mathbf{R}$ corresponde a la matriz de covarianza del sistema completo y $S_{o}$ a la intensidad del ruido blanco.

\section{Incertidumbre en los parámetros}

Dado que las matrices $\mathbf{A}_{2}$ y $\mathbf{B}_{2}$ incluyen parámetros inciertos del sistema, la matriz de covarianza de respuesta $\mathbf{R}$ también lo será. Es por ello que se puede incluir la incertidumbre de los parámetros en el cálculo de la matriz de covarianza. Para ello las matrices $\mathbf{A}_{2}, \mathbf{B}_{2}$ y $\mathbf{R}$ se aproximan en series de Taylor de primer orden, y se incluyen en (22). Donde $\overline{\mathbf{A}}_{2}, \mathbf{B}_{2}$ y $\mathbf{R}$ representan la matriz correspondiente el valor nominal del parámetro incierto (Debbarma et al., 2010). Finalmente:

$$
\begin{aligned}
& \overline{\mathbf{A}}_{\mathbf{2}} \overline{\mathbf{R}}+\overline{\mathbf{R}} \overline{\mathbf{A}}_{\mathbf{2}}^{\mathrm{T}}+\overline{\mathbf{B}}_{2} S_{o} \overline{\mathbf{B}}_{\mathbf{2}}{ }^{\mathrm{T}}=0 \\
& \overline{\mathbf{A}}_{\mathbf{2}} \frac{\partial \mathbf{R}}{\partial z_{i}}+\frac{\partial \mathbf{A}_{2}}{\partial z_{i}} \overline{\mathbf{R}}+\overline{\mathbf{R}} \frac{\partial \mathbf{A}_{\mathbf{2}}{ }^{\mathrm{T}}}{\partial z_{i}}+\frac{\partial \mathbf{R}}{\partial z_{i}} \overline{\mathbf{A}}_{\mathbf{2}}{ }^{\mathrm{T}}+\overline{\mathbf{B}}_{2} S_{o} \overline{\mathbf{B}}_{\mathbf{2}}{ }^{\mathrm{T}}=0
\end{aligned}
$$

Donde para encontrar la sensibilidad de primer orden de la matriz de covarianza $\frac{\partial \mathbf{R}}{\partial z_{i}}$ se pueden obtener resolviendo la ecuación (28). La ecuación (27) muestra las series de Taylor truncadas al primer término, de la desviación estándar de desplazamiento.

$$
\begin{gathered}
\sigma_{x_{s}}=\bar{\sigma}_{x_{s}} \pm \sum_{i=1}^{m} \frac{\partial \sigma_{x_{s}}}{\partial z_{i}} \delta z_{i} \pm \cdots, \\
\left\{\begin{array}{l}
\sigma_{x_{s u p}}=\bar{\sigma}_{x_{s}}+\sum_{i=1}^{m} \frac{\partial \sigma_{x_{s}}}{\partial z_{i}} \delta z_{i} \pm \cdots, \\
\sigma_{x_{s \text { low }}}=\bar{\sigma}_{x_{s}}-\sum_{i=1}^{m} \frac{\partial \sigma_{x_{s}}}{\partial z_{i}} \delta z_{i} \pm \cdots,
\end{array}\right.
\end{gathered}
$$

Donde:

$$
\sigma_{x_{s}}=\sqrt{R(1,1)} \quad ; \quad \frac{\partial \sigma_{x_{s}}}{\partial z_{i}}=\frac{1}{2}\left[\frac{\frac{\partial R(1,1)}{\partial z}}{\sqrt{R(1,1)}}\right]
$$

Los niveles de incertidumbre considerados en esta investigación son un 5 y 10\%. Los parámetros a analizar fueron el periodo de la estructura, razón de longitud y la razón de masa. La incertidumbre se analizará en el efecto sobre los parámetros óptimos de diseño del ACS.

\section{Procedimiento de optimización}

La optimización se realiza para tres casos, el primero para el valor real, sin incertidumbre. El segundo y tercero, corresponde al límite superior y al límite inferior del rango de incertidumbre, respectivamente. Se propone como función objetivo $\mathrm{J}$, minimizar la desviación estándar del desplazamiento de la estructura principal.

Criterio:

$J\left(\gamma_{l}, \xi_{R}, \gamma_{T}, \xi_{T}\right)=\min \left(\sigma_{x_{S}}\right)$
$J\left(\gamma_{l}, \xi_{R}, \gamma_{T}, \xi_{T}\right)=\min \left(\sigma_{x_{S} u p}\right)$ 


$$
J\left(\gamma_{l}, \xi_{R}, \gamma_{T}, \xi_{T}\right)=\min \left(\sigma_{x_{S} l o w}\right)
$$

Sujeto a:

$$
\begin{aligned}
& 0.5<\gamma_{T}<2 ; 0<\xi_{T}<1 ; 0.5<\gamma_{L}<2 ; \\
& 0<\xi_{L}<30 \\
& h-c \sigma_{y} \geq 0
\end{aligned}
$$

donde $c$ es el peak factor considerado como 2.5 en esta investigación. La restricción corresponde al límite máximo de desplazamiento en altura $h$ que puede alcanzar el líquido dentro del TLCD. $\gamma_{T}$ y $\gamma_{L}$ son las razones de sintonía que se definen en las igualdades (32a) y (32b).

$$
\gamma_{T}=\frac{\omega_{T}}{\omega_{S}} \quad ; \quad \gamma_{L}=\frac{\omega_{L}}{\omega_{S}}
$$

\section{Sensibilidad de los parámetros óptimos del ACS}

A continuación se muestran los resultados del análisis de sensibilidad de los parámetros óptimos cuando existe incertidumbre en la razón de masa $\mu$ y en la razón de longitud $p$.

\section{Incertidumbre en la razón de masa $\mu$}

Los resultados, se presentan divididos en dos figuras, una con los resultados para los parámetros óptimos correspondientes al ACLS $\left(\gamma_{L}, \xi_{l}\right)$ y otra con los de AMS $\left(\gamma_{t}, \xi_{t}\right)$. La razón de masa $\mu$, fue considerada como $50 \%$ de la razón de masa del ACLS y 50\% de la razón de masa del AMS. La Figura 2 muestra la variación de los parámetros óptimos del ACLS respecto a una incertidumbre de un 5 y $10 \%$, en forma superior e inferior. Esta figura está compuesta por 2 filas y 3 columnas. La fila superior contiene la razón de sintonía del ACLS, y la fila inferior el coeficiente de pérdida de carga. La columna de la izquierda, corresponde a un periodo de $1.5 \mathrm{~s}$, la del medio a $2.0 \mathrm{~s}$ y la de la derecha de $2.5 \mathrm{~s}$. Estos periodos representan estructuras desde una rígida a una flexible, respectivamente. Cada gráfico presenta una curva sin incertidumbre (línea azul), y las respectivas variaciones correspondientes a la incertidumbre de un 5\% (línea segmentada roja), -5\% (línea continua roja), 10\% (línea segmentada negra) y -10\% (línea continua negra).

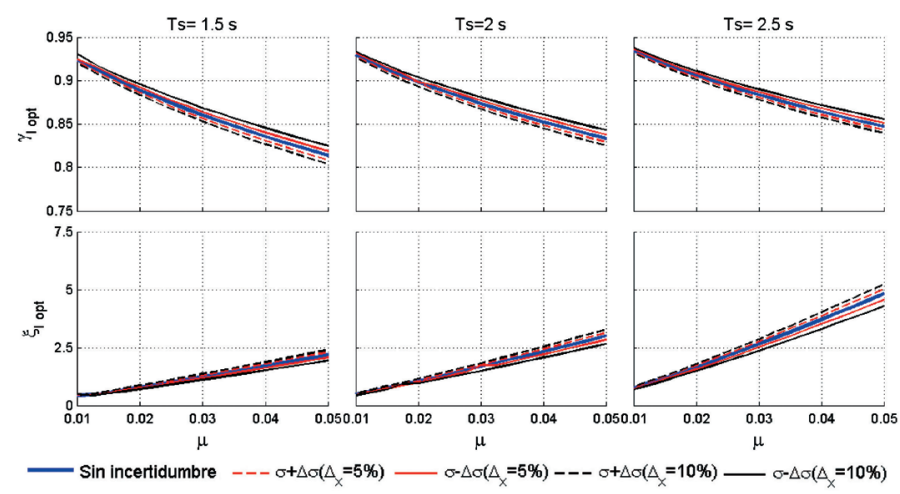

Figura 2: Parámetros óptimos del ACLS con incertidumbre de la razón de masa $\mu$

La Figura 2 muestra que a medida que aumenta la razón de masa $\mu$, la razón de sintonía $\gamma_{l}$ del ACLS disminuye, siendo levemente menor el decrecimiento, a medida que aumenta la flexibilidad de la estructura. Se observa que, para una incertidumbre negativa, la razón de sintonía es menor, y de la misma forma para una incertidumbre positiva, la razón de sintonía es mayor. Por otra parte, a medida que aumenta la razón de masa y el valor de la incertidumbre, la razón de sintonía es más sensible. Respecto al coeficiente de pérdida de carga $\xi_{l}$, se observa que a medida que aumenta la razón de masa, el coeficiente de pérdida de carga del ACLS aumenta, siendo mayor el aumento, para una estructura de mayor periodo. Se observa que, para una incertidumbre negativa, el coeficiente de pérdida de carga es mayor, y en el caso de una incertidumbre positiva, el coeficiente de pérdida de carga es menor. Por otra parte, a medida que aumenta la razón de masa, la flexibilidad de la estructura y la incertidumbre, la variación del coeficiente de pérdida de carga es mayor.

La Figura 3 muestra la variación de los parámetros óptimos del AMS respecto a una incertidumbre de un 5 y $10 \%$, en forma superior e inferior. La fila superior contiene la razón de sintonía del AMS, y la fila inferior se muestra el coeficiente de amortiguamiento del AMS. Las columnas, al igual que en la Figura 2, contienen los resultados para tres periodos, cuyos valores y distribución coinciden con los de la Figura 2. La Figura 3 muestra que a medida que aumenta la razón de masa, la razón de sintonía $\gamma_{t}$ del AMS posee el mismo comportamiento al caso de la razón de sintonía del ACLS. Sin embargo, en este caso, existe una mayor variación del parámetro óptimo cuando la estructura es más rígida. 


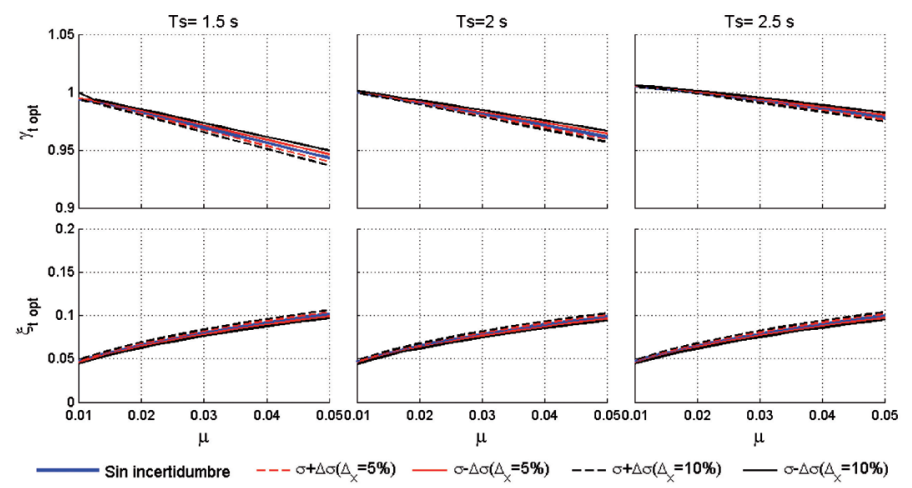

Figura 3: Parámetros óptimos del AMS con incertidumbre de la razón de masa $\mu$

Respecto a la razón de amortiguamiento óptima del AMS $\xi_{t}$, se observa que a medida que aumenta la razón de masa, el coeficiente de pérdida de carga del ACLS aumenta. Se observa que, para una incertidumbre negativa, la razón de amortiguamiento es mayor, y en el caso de una incertidumbre positiva, este es menor. Por otra parte, a medida que aumenta la razón de masa, la incertidumbre no existe una mayor variación de este parámetro óptimo.

\section{Incertidumbre en la razón de longitud $p$}

La Figura 4 muestra la variación de los parámetros óptimos del ACLS a medida que aumenta la razón de longitud, con una incertidumbre de un 5 y $10 \%$, en forma superior e inferior. Esta figura está compuesta por 2 filas y 3 columnas. La fila superior contiene la razón de sintonía del ACLS, y la fila inferior el coeficiente de pérdida de carga. Las columnas corresponden a un periodo de 1.5, 2.0 y 2.5 s. La distribución es la misma de la Figura 2. Cada gráfico presenta una curva determinística (línea azul), y las respectivas variaciones correspondientes a la incertidumbre de un 5\% (línea segmentada roja), $-5 \%$ (línea continua roja), 10\% (línea segmentada negra) y $-10 \%$ (línea continua negra).
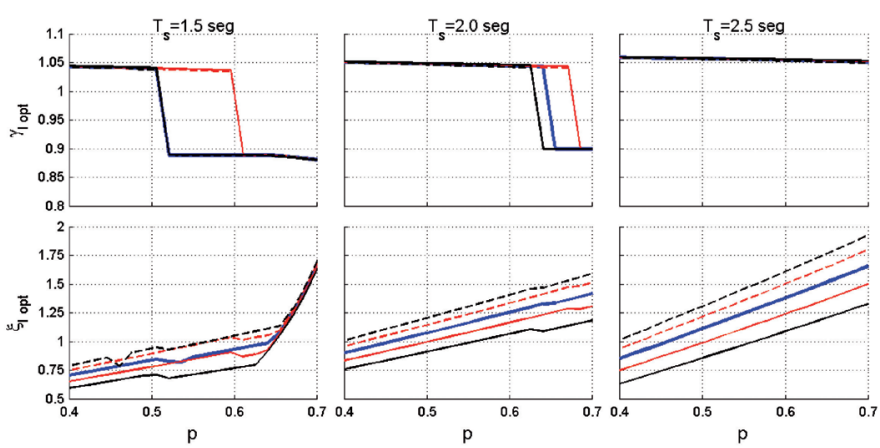

- Sin incertidumbre $---\sigma+\Delta \sigma\left(\Delta_{x}=5 \%\right)-\sigma-\Delta \sigma\left(\Delta_{x}=5 \%\right) \quad--\sigma+\Delta \sigma\left(\Delta_{x}=10 \%\right)-\sigma-\Delta \sigma\left(\Delta_{x}=10 \%\right)$

Figura 4: Parámetros óptimos del ACLS con incertidumbre de la razón de longitud $p$
La Figura 4 muestra que a medida que aumenta la razón de longitud, existe un cambio brusco en el valor de la razón de sintonía del ACLS $\gamma_{l}$. Este cambio se produce para un valor de longitud mayor, a medida que aumenta el periodo de la estructura. Además, se observa que es insensible a la incertidumbre. Respecto al coeficiente de pérdida de carga $\xi_{l}$, se observa que a medida que aumenta la razón de longitud, el coeficiente de pérdida de carga del ACLS aumenta, siendo mayor el aumento, para una estructura de mayor periodo. Se observa un quiebre en el comportamiento en este coeficiente de pérdida de carga, cambiando bruscamente su pendiente, para un valor de la razón del coeficiente de pérdida de carga mayor, a medida que es mayor la flexibilidad de la estructura. Se observa también que, para una incertidumbre negativa, el coeficiente de pérdida de carga es mayor, y en el caso de una incertidumbre positiva, el coeficiente de pérdida de carga es menor. Por otra parte, se observa que existe una gran variación de este parámetro óptimo, para los niveles de incertidumbre considerados.

La Figura 5 muestra la variación de los parámetros óptimos del AMS, respecto a una incertidumbre de 5 y $10 \%$, en forma superior e inferior. La distribución de los gráficos es la misma que en la Figura 3. Se observa que a medida que aumenta la razón de longitud, la razón de sintonía $\gamma_{t}$ del AMS disminuye. Sin embargo, existe un cambio brusco en su valor, el que se produce para un valor de longitud mayor, a medida que aumenta el periodo de la estructura. Además, se observa que es insensible a la incertidumbre. Respecto a la razón de amortiguamiento óptima del AMS $\xi_{t}$, se observa un comportamiento análogo a la sintonía óptima del AMS, pero el cambio brusco en el comportamiento del parámetro óptimo, tiene una menor magnitud.
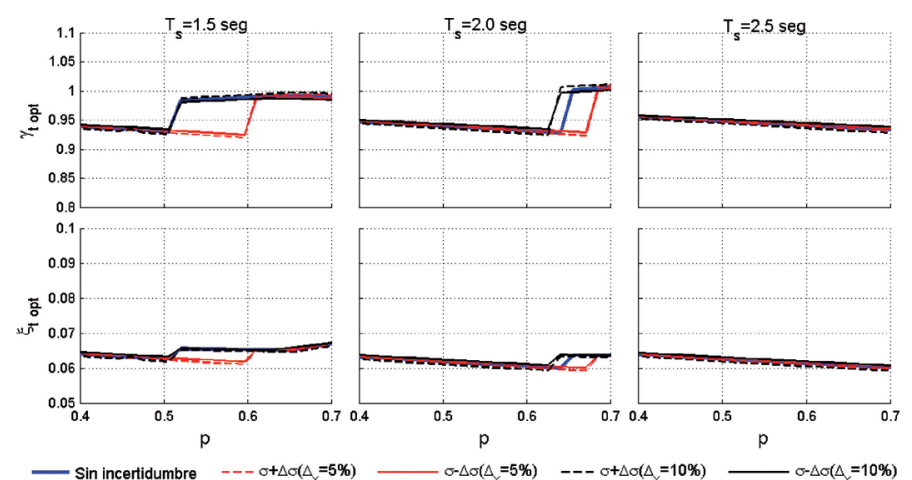

Figura 5: Parámetros óptimos del AMS con incertidumbre de la razón de longitud $p$ 
Tanto en la Figura 4 como en la Figura 5, se produce un cambio brusco en los parámetros óptimos. Esto se puede explicar a través de las ecuaciones (2a), (9b), (10d), (31) y (32b) lo que da como resultado las ecuaciones:

$$
\begin{aligned}
& h=\frac{L_{e}}{2}(1-p)=\frac{g}{\omega_{L}^{2}}(1-p)=\frac{g}{\omega_{L}^{2}}(1-p)= \\
& \frac{g}{\gamma_{L}^{2} \omega_{s}^{2}}(1-p) \geq c \sigma_{y}
\end{aligned}
$$

De donde se obtiene una constante que se muestra en la ecuación (34):

$$
f=\frac{g}{\omega_{S}^{2} c}
$$

La ecuación (35) muestra que existe una condición que involucra una discontinuidad numérica asociada a este cambio brusco. Sin embargo, se requieren estudios extras para demostrar lo obtenido en la ecuación (35).

$$
\sqrt{\frac{f(1-p)}{\sigma_{y}}} \geq \gamma_{L}
$$

\section{Sensibilidad de la eficiencia y RMS del ACS}

La eficiencia del ACS, se calcula como el porcentaje de reducción del desplazamiento (RD) del sistema principal, cuando se le adiciona un ACS óptimo.

$$
\mathrm{RD}=\left(1-\frac{\sigma_{x c / \mathrm{ACS}}}{\sigma_{x s / \mathrm{ACS}}}\right) \cdot 100 \%
$$

Donde, $\sigma_{x c / \text { ACs }}$ corresponde a la desviación estándar de desplazamiento del sistema principal controlado por un ACS óptimo adicionado y $\sigma_{x s / \text { ACS }}$ es la desviación estándar de desplazamiento del sistema principal sin ACS.

\section{Incertidumbre en la razón de masa $\mu$}

La Figura 6 muestra la eficiencia del ACS, medida como una reducción porcentual del desplazamiento de la estructura principal, es decir, mientras mayor es su valor, posee una mayor eficiencia. Las columnas muestran los resultados para 3 periodos, de $1.5,2.0$ y $2.5 \mathrm{~s}$, de izquierda a derecha, respectivamente. Los valores de incertidumbre son de un 5 y $10 \%$, en forma positiva y negativa. Se observa que a medida que aumenta la razón de masa, la eficiencia del ACS es mayor. Por otra parte, se observa que la eficiencia del ACS, ante una variación de la razón de masa de un \pm 5 $\mathrm{y} \pm 10 \%$, no sufre variación.

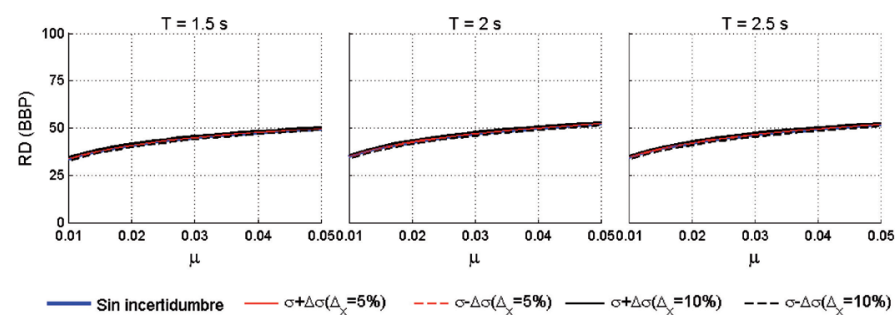

Figura 6: RD BBP eficiencia del ACS con incertidumbre de la razón de masa $\mu$

La Figura 7 muestra la respuesta media cuadrática RMS, del desplazamiento del sistema principal. Se observa que existe una leve disminución, a medida que aumenta la razón de masa. Además, se observa que a medida que la estructura tiene un mayor periodo, el RMS del desplazamiento del sistema principal es mayor. Lo que es lógico, al ser una estructura más flexible. También se observa una insensibilidad respecto a la incertidumbre en la razón de masa.

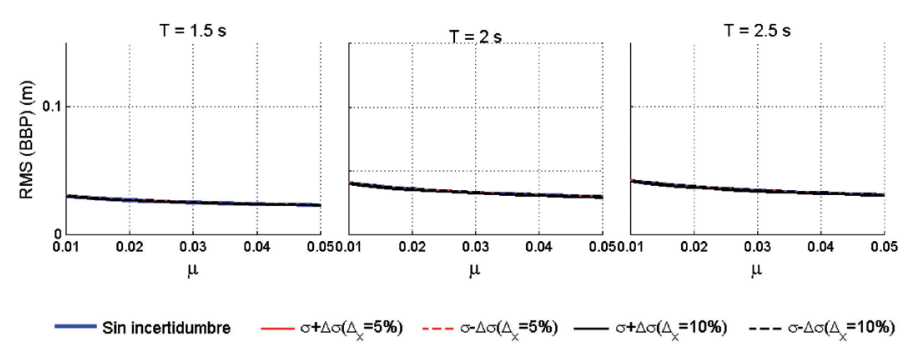

Figura 7: RMS del desplazamiento del ACS con incertidumbre de la razón de masa $\mu$

\section{Incertidumbre en la razón de longitud $p$}

La Figura 8 muestra la eficiencia del ACS, la distribución de los gráficos es la misma que en la Figura 6. Los valores de los periodos y las incertidumbres también son las mismas que en la Figura 6. Se observa que a medida que aumenta la razón de longitud, la eficiencia del ACS es mayor. Por otra parte, se observa que la eficiencia del ACS, es insensible al nivel de incertidumbre considerado en este análisis.

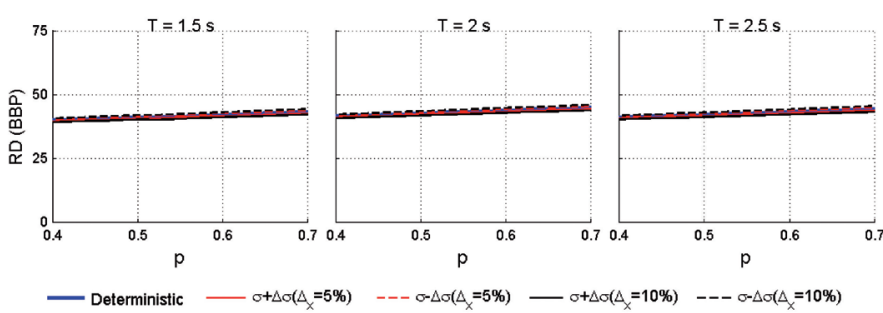

Figura 8: Eficiencia del ACS con incertidumbre de la razón de longitud $p$ 
La Figura 9 muestra la RMS del desplazamiento del sistema principal. Se observa que existe un leve aumento de este, entre un RMS para un periodo de $1.5 \mathrm{~s}$ y uno de 2.0 s. Se observa que el RMS, es independiente del valor de la razón de longitud del ACS. También se observa una insensibilidad respecto a una incertidumbre en la razón de longitud.
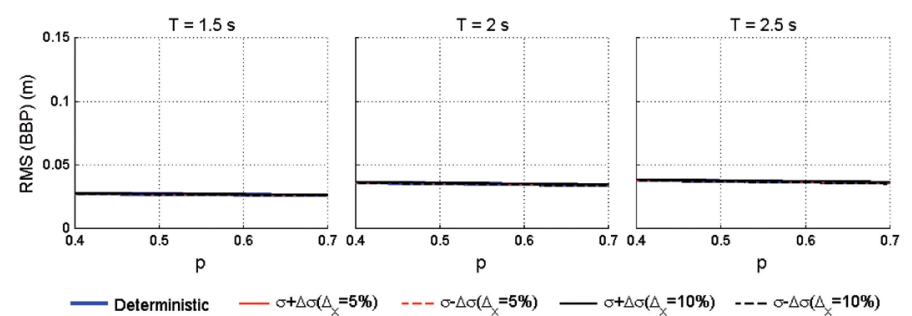

Figura 9: RMS del desplazamiento del ACS con incertidumbre de la razón de longitud $p$

\section{Conclusiones}

a. Amedida que la razón de masa es mayor, los parámetros óptimos del ACS son más sensibles a una incertidumbre en la razón de masa.

b. El único parámetro óptimo del ACS, que es sensible a una incertidumbre en la razón de longitud, es el coeficiente de pérdida de carga. El que posee una variación apreciable a medida que aumenta la razón de longitud.

c. El coeficiente pérdida de carga óptimo y la razón de amortiguamiento óptima muestran una variación positiva, ante una incertidumbre negativa.

d. La eficiencia del ACS es mayor a medida que aumenta la razón de masa, pero es prácticamente insensible a la incertidumbre.

e. La eficiencia del ACS crece levemente a medida que aumenta la razón de longitud, y es insensible a la incertidumbre.

f. El RMS es insensible a una incertidumbre en la razón de masa y una razón de longitud, pero en el primer caso, aumenta en estructuras más flexibles.

\section{Agradecimientos}

Los autores agradecen a la Universidad del Bío-Bío por el apoyo dado a través del Proyecto Regular UBB 2060542 IF/R.

\section{Referencias}

Almazán, J.L., Espinoza, G. and Aguirre, J.J. (2012). Torsional balance of asymmetric structures by means of tuned mass dampers. Engineering Structures 42, 308-328

Clough, R.W. and Penzien, J. (1975). Dynamics of structures. McGraw-Hill, USA

Debbarma, R., Chakraborty, S. and Ghosh, S. (2010). Unconditional reliability-based design of tuned liquid column dampers under stochastic earthquake load considering system parameters uncertainties. Journal of Earthquake Engineering 14(7), 970-988

Den Hartog, J.P. (1956). Mechanical vibrations. Fourth ed. Dover Civil and Mechanical Engineering, USA

Di Matteo, A., Lo Iacono, F.L., Navarra, G. and Pirrotta, A. (2015). Innovative modeling of tuned liquid column damper motion. Communications in Nonlinear Science and Numerical Simulation 23(1-3), 229-244

Di Matteo, A., Pirrotta, A. and Tumminelli, S. (2017). Combining TMD and TLCD: analytical and experimental studies. Journal of Wind Engineering and Industrial Aerodynamics 167, 101-113

Espinoza, G. Rivas, S. y Suazo, A. (2020). Análisis de la eficiencia de un amortiguador combinado sintonizado con incertidumbre en los parámetros sometido a excitaciones sísmicas de bajo contenido de frecuencias. Obras y Proyectos 28, 68-77

Espinoza, G., Almazán, J.L., Benedetti, F. and Jara, C. (2019). Torsional balance of nonlinear asymmetrical structures by means of a tuned mass damper. Structural Control and Health Monitoring 26(11), 24-42

Espinoza, G., Neira, D. y Cifuentes, A. (2018a). Análisis de un amortiguador de masa sintonizado óptimo en estructuras asimétricas no lineales. Obras y Proyectos 23, 39-54

Espinoza, G., Carrillo C. and Suazo, A. (2018b). Analysis of a tuned liquid column damper in non-linear structures subjected to seismic excitations. Latin American Journal of Solids and Structures 15(7), e91

Frahm, H. (1909). Device for damping vibrations of bodies. US patent 989,958

NCh2745 (2013). Análisis y diseño de edificios con aislación sísmica. Instituto Nacional de Normalización, Santiago, Chile 
Saitua, F., Lopez-Garcia, D. and Taflanidis, A.A. (2018). Optimization of height-wise damper distributions considering practical design issues. Engineering Structures 173, 768-786

Sakai, F. (1989). Tuned liquid column damper-new type device for suppression of building vibration. First International Conference on High-Rise Buildings, Nanjing, China, 926-931

Singh, M.P., Singh, S. and Moreschi, L.M. (2002). Tuned mass dampers for response control of torsional buildings. Earthquake Engineering and Structural Dynamics 31(4), 749-769
Wang, L., Zhao, X. and Zheng, Y.M. (2016). A combined tuned damper and an optimal design method for wind-induced vibration control for super tall buildings. The Structural Design of Tall and Special Buildings 25, 468-502

Yalla, S.K. and Kareem, A. (2000). Optimum absorber parameters for tuned liquid column dampers. Journal of Structural Engineering 126(8), 906-915 